\title{
Sarcopenia and impairment in cognitive and physical performance
}

This article was published in the following Dove Press journal:

Clinical Interventions in Aging

30 March 2015

Number of times this article has been viewed

\section{Magdalena I Tolea' \\ James E Galvin ${ }^{1-3}$}

'Alzheimer's Disease Center, Department of Neurology, ${ }^{2}$ Department of Psychiatry, ${ }^{3}$ Department of Population Health, New York University School of Medicine, New York, NY, USA

Correspondence: Magdalena I Tolea Alzheimer's Disease Center, I45 E 32nd Street, 8th Floor, New York, NY 10016 , USA

$\mathrm{Tel}+\mathrm{I} 64650 \mid 239$ |

Fax + I 2122633273

Email magdalena.tolea@nyumc.org
Background: Whether older adults with sarcopenia who underperform controls on tests of physical performance and cognition also have a higher likelihood of combined cognitive-physical impairment is not clear. We assessed the impact of sarcopenia on impairment in both aspects of functionality and the relative contribution of its components, muscle mass and strength.

Methods: Two hundred and twenty-three community-dwelling adults aged 40 years and older (mean age $=68.1 \pm 10.6$ years; $65 \%$ female) were recruited and underwent physical functionality, anthropometry, and cognitive testing. Participants with low muscle mass were categorized as pre-sarcopenic; those with low muscle mass and muscle strength as sarcopenic; those with higher muscle mass and low muscle strength only were categorized as non-sarcopenic and were compared on risk of cognitive impairment (Montreal Cognitive Assessment $<26$; Ascertaining Dementia $8 \geq 2$ ), physical impairment (Mini Physical Performance Test $<12$ ), both, or neither by ordinal logistic regression.

Results: Compared to controls, those with sarcopenia were six times more likely to have combined cognitive impairment/physical impairment with a fully adjusted model showing a three-fold increased odds ratio. The results were consistent across different measures of global cognition (odds ratio $=3.46,95 \%$ confidence interval $=1.07-11.45$ for the Montreal Cognitive Assessment; odds ratio $=3.61,95 \%$ confidence interval $=1.11-11.72$ for Ascertaining Dementia 8). Pre-sarcopenic participants were not different from controls. The effect of sarcopenia on cognition is related to low muscle strength rather than low muscle mass.

Conclusion: Individuals with sarcopenia are not only more likely to have single but also to have dual impairment in cognitive and physical function. Interventions designed to prevent sarcopenia and improve muscle strength may help reduce the burden of cognitive and physical impairments of functionality in community-dwelling seniors.

Keywords: cross-sectional, muscle strength, muscle mass, physical impairment, cognitive impairment, older adults

\section{Introduction}

Development of disability most commonly starts with an underlying pathologic process (eg, reduction in type II muscle fiber size) resulting in a measurable impairment (eg, reduced muscle mass and strength), and functional limitation (eg, reduced mobility and balance) ultimately resulting in some form of disability (eg, interference with activities of daily living [ADL] ). ${ }^{1}$ Approximately 23.7 million older Americans reported difficulty performing $\geq 1$ ADL (basic or instrumental) in 2011, making disability a very common, chronic condition affecting approximately three out of five adults aged $\geq 65$ years. ${ }^{2}$ Disability also increases likelihood of falls ${ }^{3}$ and institutionalization, ${ }^{4}$ has a negative impact on quality of life, ${ }^{5}$ and increases mortality risk. ${ }^{6}$

Although physical difficulties can occur independently of cognitive decline, in many seniors, physical difficulties coexist with cognitive impairment (CI). Behavioral 
Risk Factor Surveillance System 2011 data suggest that CI is present in $12.7 \%$ of individuals aged $\geq 60$ years. ${ }^{7}$ Of these, $35.2 \%$ also report physical functional difficulties. Having both cognitive and physical functional impairment may be particularly taxing on the affected individuals and their caregivers. However, how cognitive and functional decline are linked and the factors that may be involved are still unclear. Identification of early markers of dual decline is therefore an important research goal.

Sarcopenia, or reduced muscle mass and function, may represent one such factor. Low muscle mass and muscle strength have each been linked to higher levels of physical limitation and disability, ${ }^{8} \mathrm{CI} /$ dementia, ${ }^{9-11}$ and brain atrophy. ${ }^{12}$ While the role sarcopenia plays in the processes that leads to physical decline is generally understood and supported empirically, its relation with cognitive dysfunction is less clear. Although there is general consensus that motor neuronal dysfunction can lead to decrements in muscle mass and strength, a reverse association via decline in physical activity participation or a common underlying process cannot be ruled out, ${ }^{13,14}$ suggesting a complex interplay between physical and cognitive decline in which sarcopenia may play an important role.

These individual associations suggest that sarcopenia may also be involved in the processes that leads to combined physical-cognitive dysfunction, with potential implications for interventions targeting older adults at risk of developing both types of impairment. Moreover, since sarcopenia is a complex concept involving both low muscle mass and muscle function, a differential effect of these components on physical and cognitive functionality in later life is possible, with strength being the more consistently reported predictor of future disability. ${ }^{8}$

The goal of our study is two-fold: 1) to evaluate the association between sarcopenia and CI, physical impairment (PI) or dual impairment (CI/PI); and 2) to investigate whether the sarcopenia effect is driven by muscle mass, muscle strength, or both. We expected that sarcopenia would be associated with an increased likelihood of combined dysfunction and that muscle strength would be the most influential component.

\section{Methods}

Participants were community-dwelling adults aged 40 years and older recruited to participate in research projects studying 1) the use of screening tests to detect CI and PI in a multicultural community, and 2) corresponding biomarkers of cognitive decline at New York University (NYU), Langone Medical Center between February 2012 and January 2014.
One study (NIH Grant 5R01AG040211-02 Galvin, unpublished data, 2015) focused on medical and cognitive screening and biomarkers in older adults (age 65+ years) while a second study (Schapiro Fund, unpublished data, 2015) used the same protocol in middle-aged adults (age $40+$ years). The third study (Fox Foundation, unpublished data, 2015) using the same protocol recruited a sample of healthy controls (age $50+$ years) for comparison to individuals with Parkinson's disease. For all three studies, participants were recruited via word-of-mouth, solicitation at educational seminars on dementia and other age-related conditions, collaborations with local senior centers, housing projects, churches, and other organizations advocating for the betterment of seniors, particularly minorities and other disadvantaged groups, and advertisements to individuals registered into the Research Match database maintained by NYU Clinical Science and Translational Institute. The study protocol and procedures were approved by the NYU Institutional Review Board. Written informed consent was obtained from each participant.

Potential participants were given an Institutional Review Board-approved study information sheet explaining the project objectives, and inclusion and exclusion criteria. Inclusion criteria included age $\geq 40$ years and either English- or Spanishspeaking status. Exclusion criteria included age $<40$ years, primary language other than English or Spanish, established diagnosis of a primary Axis I psychiatric disorder (eg, schizophrenia, bipolar disease), or degenerative neurologic disorder (ie, Parkinson's disease, amyotrophic lateral sclerosis) that could impact cognitive and/or physical performance or cooperation with study procedures. No other exclusions were made on the basis of medical, psychiatric, or social conditions, as the goal of this part of the first aforementioned study (Galvin, unpublished data, 2015) was to determine the effectiveness, utility, willingness, acceptance, preferences, and compliance with dementia screening. Eligible individuals were offered the option of conducting the research visit at their local senior center/institution through which they were recruited or to come to the NYU Langone Medical Center.

A total of 241 participants had a clinic visit during which socio-demographic, financial, cognitive, physical, emotional, and functional health testing was performed using standardized assessment tools, and a detailed medical history was obtained. Of these, 223 (93\%) had valid data on muscle mass and strength and were therefore included in this study.

\section{Outcome variables}

Cognitive function was measured using two validated methods for detecting cognitive impairment in older 
adults. The Montreal Cognitive Assessment (MoCA) ${ }^{15}$ is a performance-based brief assessment of visuospatial skills, executive function, attention, language, memory recall, and orientation. A total score (range, 0-30 points) is calculated by summing up individual scores and with lower scores representing poorer cognitive performance. In community samples, a cut-off score of $<26$ points is suggested to indicate impaired cognitive function. To account for educational differences, an extra point is added for those with $\leq 12$ years of education.

The Ascertaining Dementia 8 (AD8) is an eight-item, yes/no answer, patient-reported assessment (range, 0-8 points) that identifies early cognitive changes compared to previous abilities ${ }^{16}$ in memory, problem-solving, orientation, and daily activities. Originally designed as an informantbased assessment, the AD8 has been validated as a selfreported measure of cognitive change. ${ }^{17}$ The AD8 correlates highly with Alzheimer's disease spinal fluid and imaging biomarkers. ${ }^{18}$ Higher scores imply greater likelihood of cognitive impairment, and a cut-off score $\geq 2$ points is used to indicate impaired cognitive function.

\section{Physical function}

Physical function was measured using the Mini Physical Performance Test (MPPT), ${ }^{19}$ which includes the following tasks: pick-up-penny (a bending-over task), 50-foot usual-pace walking test, five complete chair-raises, and the progressive Romberg balance test, each ranging from 0 to 4 points with a score of 4 points indicating highest level of performance. The MPPT was derived from a regression analysis of the longer Physical Performance Test ${ }^{20}$ and was tested in a longitudinal sample of older adults with and without dementia. Changes in the MPPT scores correlated with the development of disability, the loss of independence, the risk of falls, and mortality. ${ }^{19}$ The MPPT was derived from the same scale as the Short Portable Performance Battery ${ }^{21}$ but includes a bending-over task, as these type of activities are commonly associated with falls in older adults. A total MPPT score (range, 0-16 points) is calculated by summing up scores for the four individual tasks, with lower scores implying worse physical functioning. A cut-off score of $<12$ points is used to indicate impaired physical function.

\section{Cognitive-physical classifications}

Using each of the two cognitive measures separately (ie, MoCA and AD8) and the physical functional test (ie, MPPT), participants were categorized as having no impairment, either CI or PI, or combined CI/PI. This classification was made to test whether patient-reported CI vs performance testing had different relationships to physical function.

\section{Anthropometric measurements}

Total muscle mass was derived from bioelectrical impedance analysis with the BC-558 Ironman Segmental Body Composition Monitor (Tanita Corporation, Arlington Heights, IL, USA) and expressed in pounds (lb). Impendence measurements of muscle mass using Tanita scales have $90 \%$ correlation with dual energy X-ray absorptiometry. ${ }^{22}$ Handgrip strength was measured with a handheld dynamometer (Baseline ${ }^{\circledR}$ Digital Smedley Spring Dynamometer; Patterson Medical, Warrenville, IL, USA) and expressed in lb. One measurement was taken for each hand using maximal effort, and the mean of the two measurements was used in data analysis. Due to its correlation to other strength measures, handgrip strength is often used in aging studies as an indicator of overall muscle strength. ${ }^{23}$

\section{Predictor variable}

The concept of sarcopenia was first proposed in 1989 by Irwin Rosenberg to describe the loss of skeletal muscle mass with aging, ${ }^{24}$ and guidelines for defining sarcopenia based on muscle mass were proposed. ${ }^{25}$ Loss of muscle function as measured by muscle strength has since been added to the definition of sarcopenia. ${ }^{26}$ More recent guidelines suggest low physical performance as another alternative measure of muscle dysfunction (either low muscle strength or low physical performance), although presence of low muscle mass remains an essential diagnosis criterion. ${ }^{27}$ Other measures of sarcopenia include the Short Portable Sarcopenic Measure consisting of lean body mass index (BMI), grip strength, and lower extremity strength (timed chair rises). ${ }^{22}$ However, the inclusion of poor physical performance (eg, gait speed) in the definition of sarcopenia has been questioned, as its effectiveness in identifying sarcopenic cases is similar to that of the muscle mass and strength algorithm. ${ }^{28}$ Therefore, for the purpose of this study, we categorized participants into the following groups: non-sarcopenic (high muscle mass and high muscle strength), pre-sarcopenic (low muscle mass and high muscle strength), and sarcopenic (low muscle mass and low muscle strength). Since low muscle mass is a required component of sarcopenia, and current guidelines do not include low muscle strength alone as evidence of presarcopenia, ${ }^{27}$ participants with low strength but high mass were included in the non-sarcopenia group. For the purpose 
of this study, low muscle mass and strength were defined as being in the bottom quartile of the sample distribution.

\section{Covariates}

Increased age, female sex, minority status (White vs nonWhite), BMI; measured as weight/height ${ }^{2}$ are significant correlates of CI, PI, and/or sarcopenia, and were included as potential covariates in data analysis. Depressive symptoms were measured with the Hospital Anxiety and Depression Scale $^{29}$ and were included as a covariate.

\section{Data analysis}

Mean differences in the variables of interest among the three outcome groups (no impairment; CI or PI; combined CI/PI) were analyzed with analysis of variance and chi-square tests (Table 1). We then employed a series of ordinal logistic regression models to investigate the effect of sarcopenia on the likelihood of combined CI/PI (Table 2). The first model tests the unadjusted effect of sarcopenia on the outcome, the second model adds age, sex, race, and BMI to the equation, and the third model additionally includes depression. The Akaike Information Criterion was used to identify the model that best fits the data using a score of $>4$ as the cut-off point for both MoCA and AD8. ${ }^{30}$ All models satisfied the proportional odds assumption. The proportional odds ratio that results from ordinal logistic models is interpreted as odds of having both types of impairment against the cumulative odds of having either one impairment or none associated with one unit increase in the predictor (ie, pre-sarcopenia vs controls; sarcopenia vs controls). Results are presented separately for MoCA and AD8, the two measures of cognitive function investigated in this study. In order to investigate whether the inclusion of low muscle strength only in the non-sarcopenia group would have an impact on our results, we conducted a sensitivity analysis in which low muscle strength alone was included as a separate group. Last, in order to determine which component of sarcopenia (muscle mass or strength) drives the association with impairment, we modeled the effect of having low vs higher muscle mass and strength, respectively, on the odds of combined CI/PI adjusted for age, sex, race, BMI, and depression (Table 3). Data analysis was performed using the SAS system version 9.2 (SAS Institute Inc, Cary, NC, USA) and a $P$-value of $<0.05$ to indicate statistical significance.

\section{Results}

As Table 1 indicates, compared to participants with either none or one type of impairment, CI/PI participants were older, less educated, more likely to be female and non-White, had lower muscle mass and strength, took longer to walk 50 feet, and had poorer cognitive performance on both MoCA and AD8. The three groups also differed in terms of level of comorbidity, as measured by the Charlson Comorbidity Index, with higher comorbidity in dually impaired participants than in those with either type of impairment or none $(2.5 \pm 1.4$ vs $1.9 \pm 1.3$ and $0.9 \pm 1.2$, respectively; overall $P<0.001$ ). In addition, when using AD8 to measure cognitive abilities, a dose-response association was found between depression and type of impairment, with a mean depression score of 3.7 in those with no impairment, 6.1 in those with either type of impairment, and 7.5 in the dually impaired group.

The proportion of participants in each of the three outcome groups by sarcopenia category is presented in Figure 1.

Table I Participant characteristics by level of impairment

\begin{tabular}{|c|c|c|c|c|c|c|c|c|}
\hline \multirow[t]{2}{*}{ Characteristic } & \multicolumn{4}{|c|}{$\begin{array}{l}\text { Cognitive impairment }(\text { MoCA }<26) \text { and physical } \\
\text { impairment }\end{array}$} & \multicolumn{4}{|c|}{$\begin{array}{l}\text { Cognitive impairment }(A D 8 \geq 2) \text { and physical } \\
\text { impairment }\end{array}$} \\
\hline & None & Either type & Both types & $P$-value & None & Either type & Both types & $P$-value \\
\hline Age, yrs & $62.9( \pm 9.7)$ & $66.5( \pm 10.3)$ & $74.3( \pm 7.6)$ & $<0.001$ & $62.8( \pm 9.8)$ & $69.2( \pm 9.2)$ & $75.4( \pm 9.4)$ & $<0.001$ \\
\hline Education, yrs & $14.8( \pm 3.2)$ & $14.2( \pm 3.9)$ & $10.8( \pm 4.7)$ & $<0.001$ & $14.7( \pm 3.7)$ & $13.2( \pm 4.4)$ & $10.7( \pm 4.8)$ & $<0.001$ \\
\hline Female, \% & 62.7 & 55.9 & 81.8 & 0.005 & 55.9 & 64.4 & 85.0 & 0.006 \\
\hline White race, \% & 60.3 & 39.0 & 25.9 & 0.006 & 50.6 & 36.1 & 28.2 & 0.108 \\
\hline $\mathrm{BMI}, \mathrm{lb} / \mathrm{in}^{2}$ & $27.6( \pm 6.2)$ & $27.8( \pm 5.3)$ & $29.2( \pm 5.3)$ & 0.278 & $27.9( \pm 6.0)$ & $28.2( \pm 5.5)$ & $28.1( \pm 5.1)$ & 0.939 \\
\hline Depression & $4.6( \pm 3.6)$ & $5.4( \pm 4.07)$ & $5.9( \pm 4.21)$ & 0.256 & $3.7( \pm 2.9)$ & $6.1( \pm 3.9)$ & $7.5( \pm 4.8)$ & $<0.001$ \\
\hline Muscle mass, lb & $106.4( \pm 24.7)$ & $105.8( \pm 22.9)$ & $91.6( \pm 22.1)$ & $<0.001$ & $107.6( \pm 25.0)$ & $102.3(9 \pm 20.8)$ & $89.4( \pm 22.8)$ & $<0.001$ \\
\hline Grip strength, lb & $64.3( \pm 26.7)$ & $58.7( \pm 24.9)$ & $42.3( \pm \mid 3.6)$ & $<0.001$ & $66.4( \pm 27.3)$ & $51.1( \pm 19.9)$ & $41.3( \pm 12.9)$ & $<0.001$ \\
\hline Walking time, sec & $13.6( \pm 2.2)$ & $14.8( \pm 3.9)$ & $20.1( \pm 4.2)$ & $<0.001$ & $13.5( \pm 2.0)$ & $16.4( \pm 4.7)$ & $20.5( \pm 5.1)$ & $<0.001$ \\
\hline MoCA score, $0-30$ points & $27.8( \pm I .3)$ & $21.9( \pm 4.9)$ & $19.4( \pm 4.2)$ & $<0.001$ & $24.5( \pm 4.5)$ & $22.1( \pm 5.6)$ & $21.2( \pm 4.6)$ & $<0.001$ \\
\hline AD8 score, $0-8$ points & I.I ( $\pm \mathrm{I} .8)$ & $1.8( \pm 1.9)$ & $2.0( \pm 1.8)$ & 0.012 & $0.3( \pm 0.5)$ & $2.4( \pm 2.1)$ & $3.4( \pm I .2)$ & $<0.001$ \\
\hline
\end{tabular}

Notes: Physical impairment = MPPT $<12$ points; depression was measured with HADS. All data are \pm SD unless otherwise noted.

Abbreviations: yrs, years; lb, pounds; sec, seconds; in, inches; SD, standard deviation; BMI, Body Mass Index; MoCA, Montreal Cognitive Assessment; MPPT, Mini Physical Performance Test; HADS, Hospital Anxiety Depression Scale; AD8, Ascertaining Dementia 8. 
Table 2 Likelihood of having both cognitive and physical functional impairment (vs either type of impairment or none), according to level of sarcopenia

\begin{tabular}{|c|c|c|c|c|c|c|}
\hline \multirow[t]{2}{*}{$\begin{array}{l}\text { Level of } \\
\text { sarcopenia }\end{array}$} & \multicolumn{3}{|c|}{$\begin{array}{l}\text { OR of having both cognitive impairment (based } \\
\text { on MoCA) and physical impairment }{ }^{\epsilon}\end{array}$} & \multicolumn{3}{|c|}{$\begin{array}{l}\text { OR of having both cognitive impairment (based } \\
\text { on AD8) and physical impairment }\end{array}$} \\
\hline & Unadjusted & Adjusted I & Adjusted 2 & Unadjusted & Adjusted I & Adjusted 2 \\
\hline Controls & 1.0 & 1.0 & 1.0 & 1.0 & 1.0 & 1.0 \\
\hline Pre-sarcopenia & $0.90(0.426-1.940)$ & $1.09(0.415-3.853)$ & I.54 (0.544-4.370) & $0.93(0.432-1.986)$ & $0.80(0.302-2.137)$ & I. $10(0.375-3.207)$ \\
\hline \multirow[t]{2}{*}{ Sarcopenia } & 6.02 & 4.09 & 3.46 & 6.10 & 3.07 & 3.612 \\
\hline & $(2.577-|4.33|)$ & (I.405-II.907) & (I.072-II.452) & $(2.729-14.068)$ & $(1.098-8.607)$ & $(1.113-11.725)$ \\
\hline$N$ & 213 & 196 & 180 & 217 & 200 & 184 \\
\hline AIC & 438.3 & 376.9 & 345.7 & 439.5 & 366.6 & 309.0 \\
\hline
\end{tabular}

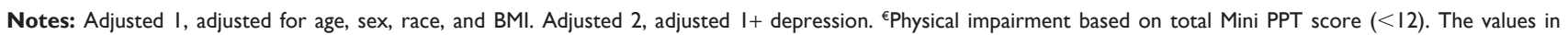
parentheses represent $95 \%$ confidence intervals.

Abbreviations: N, number of subjects; AIC, Akaike Information Criterion; OR, odds ratio; MoCA, Montreal Cognitive Assessment; AD8, Ascertaining Dementia 8; vs, versus.

While nearly one in three of those without evidence of sarcopenia had neither CI (based on MoCA) nor PI, only one in 12 sarcopenic participants had no impairment. Similarly, $20 \%$ of controls had combined CI/PI while the proportion was three times higher in those with sarcopenia. Pre-sarcopenic participants fell between controls and sarcopenic participants. This stepwise association is further evidenced in Table 2, in which the odds of being dually impaired vs singly impaired or non-impaired were $10 \%-50 \%$ higher in the pre-sarcopenia group (depending on the cognitive measure used) but $250 \%$ higher in the sarcopenia group in the fully adjusted model. Statistical significance was achieved for sarcopenia but not for pre-sarcopenia ( $P=0.415$ for MoCA; $P=0.867$ for AD8). Akaike Information Criterion scores indicate the fully adjusted model as best fitting the data. ${ }^{30}$ Our sensitivity analysis indicates that inclusion of low muscle strength only in the non-sarcopenia group did not impact our result. The effect of pre-sarcopenia and sarcopenia on the likelihood of combined $\mathrm{CI} / \mathrm{PI}$ remained unchanged (odds ratio $[\mathrm{OR}]=1.353, P=0.590$ $\mathrm{OR}=1.568, P=0.406$ for pre-sarcopenia vs non-sarcopenia using AD8 and MOCA, respectively; $\mathrm{OR}=4.658, P=0.013$ and $\mathrm{OR}=3.574, P=0.037$ for sarcopenia vs non-sarcopenia using AD8 and MOCA, respectively).

Lastly, we investigated the individual effect of low muscle mass and strength on the outcome (Table 3). The fully adjusted models using MoCA scoring $<26$ points for CI show no significant individual effect of either low muscle mass or low strength, although the effect sizes indicate at least a $50 \%$ higher odds of combined CI/PI associated with both sarcopenia components. The AD8 measure, however, indicates a significant effect for muscle strength, with the group scoring in the bottom quartile of strength being almost three times more likely to have combined CI/PI than either one impairment or none compared to their higher muscle strength counterparts. No effect was seen for muscle mass $(P=0.13)$.

\section{Discussion}

In this cross-sectional analysis of a large sample of community-dwelling older adults from diverse backgrounds, we found that sarcopenia, defined as low muscle mass and muscle strength, is associated with having a combination of $\mathrm{CI}$ and PI. Low muscle mass, defined in this study as presarcopenia stage, does not appear to increase the likelihood of combined CI/PI when compared to high muscle mass and strength, suggesting that the increased link with sarcopenia likely comes from having low muscle strength.

More than 2.6 million adults aged 60 years and older reported CI accompanied by physical functional impairment in 2011. ${ }^{7}$ For these individuals and their families, finding ways to improve quality of life is of paramount importance. Moreover, understanding the correlates of combined CI/PI may lead to preventative programs and is therefore of great importance from a public health perspective. While poor cognitive and functional performance and higher levels of ADL limitations, particularly with complex tasks have been identified, are important predictors of combined CI/PI, ${ }^{8}$ a focus on factors that occur earlier in the impairment process is likely a better approach to prevention.

Table 3 Individual effect of muscle mass and muscle strength on cognitive and functional impairment

\begin{tabular}{lll}
\hline Predictor & OR of combined impairment (MoCA) & OR of combined impairment (AD8) \\
\hline Low vs normal/high MM & $2.290(0.970-5.407)$ & I.946 (0.8I6-4.639) \\
Low vs normal/high grip strength & $1.498(0.708-3.172)$ & $2.673(1.213-5.627)$ \\
\hline
\end{tabular}

Notes: ${ }^{\epsilon}$ Adjusted for age, sex, race, BMI, and depression. The values in parentheses represent $95 \%$ confidence intervals.

Abbreviations: BMI, body mass index; MoCA, Montreal Cognitive Assessment; MM, muscle mass; vs, versus; OR, odds ratio; AD8, Ascertaining Dementia 8. 


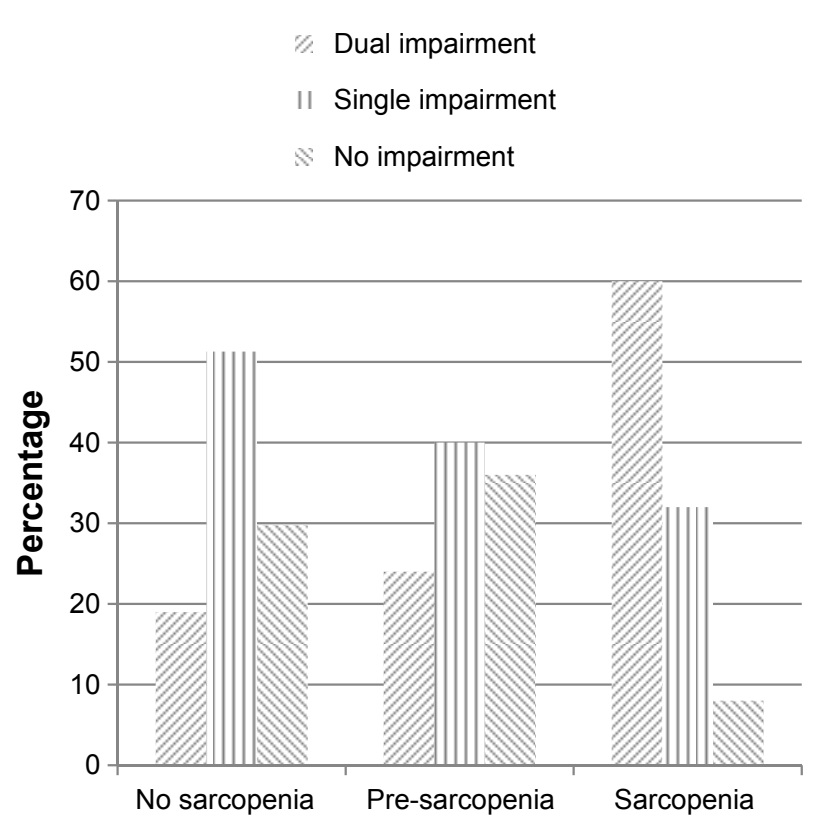

Figure I Distribution of impairment by sarcopenia status. Notes: Controls have lowest level of dual impairment, followed by pre-sarcopenic and finally, sarcopenic participants. Differences are significant at $P<0.001$.

Sarcopenia captures early-stage changes in muscles that can affect one's physical functionality either at the personlevel (eg, locomotion, balance) or the more socially-integrated level of being able to fulfill social roles considered normal for an older person (eg, ADL, hobbies). For example, in large samples of senior populations, low muscle mass and strength have been associated with a higher likelihood of both selfreported and objectively measured physical limitation and disability ${ }^{9,31}$ as well as brain atrophy ${ }^{12}$ and dementia. ${ }^{32}$

Our combined CI/PI measure (with three levels: no impairment, CI or PI, and combined $\mathrm{CI} / \mathrm{PI}$ ) required ordinal logistic regression modeling in which the likelihood of combined impairment is estimated against the cumulative likelihood of either impairment or none, therefore making it difficult to distinguish the effect of sarcopenia on the two individual types of impairment. However, our findings point to sarcopenia as being an important correlate of $\mathrm{CI} / \mathrm{PI}$ and therefore a potentially important factor to consider in our efforts to identify individuals at risk of developing both disability and dementia. The effect is substantial and robust, holding across different measures of cognition and after adjustment for significant correlates of both CI and PI. This supports previous reports of associations between sarcopenia or its components and individual functional outcomes, such as physical and cognitive function. For example, in a large sample of participants in the Third National Health and Nutrition Examination Survey, sarcopenia measured as low skeletal muscle mass was linked to functional impairment and disability after adjustment for age, race, BMI, health behaviors, and comorbidity. ${ }^{31}$ In another large epidemiologic study of health and aging, low grip strength defined as being in the lowest $20 \%$ of the sample distribution was associated with physical limitation and instrumental activities of daily living disability. ${ }^{9}$ A lower level of grip strength was also reported to increase the risk of Alzheimer's disease in cognitively normal older adults. ${ }^{32}$ Although we are unaware of any reports on an association with combined CI/PI, our finding suggests that older adults on route to developing physical disabilities and/ or dementia, as evidenced by lower cognitive and physical performance, may be targeted for interventions to maintain function and delay/prevent impairment/disability as soon as they exhibit signs of decreased muscle function.

Pre-sarcopenia, defined as low muscle mass in accordance with current guidelines, ${ }^{27}$ did not appear to increase likelihood of combined CI/PI compared to having normal mass and strength. This could be interpreted as suggesting that low muscle mass by itself is not a significant contributor. Since our sarcopenia categorization scheme does not allow direct investigation of the individual effect of the two components of sarcopenia, we ran separate ordinal logistic regression models contrasting low and higher muscle mass and strength in terms of likelihood of combined CI/PI. In fully adjusted models, we found evidence that muscle strength is likely the component driving the effect of sarcopenia. This finding supports recent reports of a stronger impact of strength on physical and cognitive functionality compared to muscle mass. ${ }^{9,11,32,33}$ For example, in a cross-sectional analysis of a large cohort of elderly Australian men, measures of upper and lower extremity muscle strength were stronger predictors of PI and disability compared to either muscle mass or muscle quality. ${ }^{9}$ Similarly, studies investigating the differential effect on cognition suggest superiority of muscle strength as a correlate/predictor of cognitive functionality. ${ }^{11,32,33}$ This result is expected, as age-related strength loss appears to surpass the age-related loss of muscle mass, ${ }^{34}$ and the former is not entirely explained by the latter. ${ }^{35}$ Recently published evidence from the Foundation for the National Institutes of Health (FNIH) Biomarkers Consortium Sarcopenia Project, pooling data from nine large international studies of community-dwelling older adults ( $\mathrm{N}=26,625$ adults), suggests that non-mass pathways to low muscle strength and poor physical function are possible and that low muscle mass may be a significant contributor to poor physical function only when accompanied by weakness. ${ }^{36}$ This provides further evidence that low strength is likely the strongest contributor to poor functionality. 
Although lower extremity strength is more relevant for gait and balance, handgrip strength is highly correlated to other measures of muscle strength ${ }^{37}$ and can therefore be used as a good indicator of overall muscle strength. In addition, it can be easily and inexpensively measured in geriatric practice and can help identify those at increased risk for negative health outcomes including disability, falls, and frailty. ${ }^{38}$ Our findings indicate that handgrip strength can also be used as a marker for concurrent CI and PI. Prospective studies are needed to confirm that the observed cross-sectional associations operate longitudinally, and to help establish a causal link between sarcopenia and low muscle strength in particular, and development of comorbid CI/PI.

Several mechanisms could explain the link between sarcopenia and low muscle strength and dual CI/PI. Declines in muscle mass and strength resulting from normal aging (eg, age-related muscle fiber atrophy ${ }^{39}$ and decline in muscle mitochondrial function ${ }^{40}$ ), secondary events (eg, acute trauma) or disease-related processes (eg, myopathy, muscular dystrophy) can lead to physical limitations and disability, even in the absence of evident CI. The coexistence of $\mathrm{CI}$ and PI in some older adults, however, suggests a close link between these two processes, although at the present time this interconnectedness is poorly understood. Based on evidence of greater decline in muscle strength in older adults with poor cognition, ${ }^{41}$ one likely pathway involves $\mathrm{CI}$ as a precedent of muscle wasting and PI. CI at its earliest stages may lead to physical inactivity, which in turn leads to muscle mass and strength loss and therefore to poor physical performance. ${ }^{42}$ In addition, loss of motor neurons can lead to loss of motor units, which in the context of age-related reductions in neuron-muscle fiber re-innervation processes ${ }^{43}$ translates into muscle atrophy and wasting, and subsequent physical decline. However, recent evidence of increased risk of Alzheimer's disease in cognitively normal but mildly physically impaired older individuals suggests that physical decline may also precede and lead to cognitive decline, ${ }^{44}$ possibly through a reduction in level of participation in physical activities, which negatively impacts cognition. Finally, cognitive and physical decline could share common underlying processes, such as low-level chronic inflammation, oxidative stress, and depression. The skeletal muscle can produce and secrete interleukin-1, interleukin-6, and other important myokines ${ }^{45}$ which in turn have been implicated in cognitive and physical functional degenerative processes. ${ }^{46,47}$ Oxidative stress has been linked to sarcopenia ${ }^{48}$ and $\mathrm{PI},{ }^{49}$ and has also been found to be involved in neuronal degeneration and $\mathrm{CI} .{ }^{50}$ Associations between CI, depression, and sarcopenia have also been reported..$^{51}$ In our study, depression was not a significant mediator of the association of sarcopenia and combined CI/PI, reducing the effect of sarcopenia on the outcome based on the performance measure of cognition but not when based on the self-reported measure of cognition (Table 2). This result suggests that other mechanisms including those described in this section, may play a bigger role in these associations.

These results should be discussed keeping in mind limitations related to our study. Due to its cross-sectional design, we are unable to establish directionality in the associations between sarcopenia and dual CI/PI. Longitudinal studies are needed, as they will indicate whether interventions to prevent development of comorbid CI/PI would be successful if designed to address muscle strength loss. Also, our choice of muscle mass measure (ie, absolute mass) may have reduced our ability to detect an association with muscle mass. Relative (to BMI), but not absolute, muscle mass has been reported as a potentially significant contributor to development of mobility impairment in previous studies. ${ }^{31,52}$ However, since we did adjust for the effect of BMI, we believe that the likelihood of this threat is relatively low and that our results could be interpreted as indicating that low muscle mass that is not associated with low muscle strength may be a non-important threat to functionality. Strengths of our study include the relatively large sample size, representativeness of our sample, and use of standardized instruments to measure muscle mass, muscle strength, and physical and cognitive function.

In conclusion, our findings suggest that sarcopenia and its strength component in particular, may be used as an indicator for combined CI/PI, which when present suggests a risk for development of functional limitations and disability and/or dementia. Given the success reported for physical activity interventions in improving physical and cognitive performance, even among individuals with sarcopenia, ${ }^{53}$ whether working by improving strength via increasing muscle mass or through other pathways, ${ }^{36}$ such interventions have the potential to delay development of physical disability and dementia, and therefore address an important public health concern.

\section{Acknowledgments}

This work was supported by the National Institutes of Health (grant 5R01AG040211-02), the Michael J Fox Foundation for Parkinson's Research, and the Morris and Alma Schapiro Foundation.

\section{Author contributions}

Both Dr Tolea and Dr Galvin had full access to all data in the study and made substantial contributions to conception and 
design, acquisition of data, or analysis and interpretation of data; Dr Tolea drafted the manuscript and both authors provided critical revisions of intellectual content; Both authors give final approval of the version to be published; and both authors are accountable for all aspects of the work in ensuring that questions related to the accuracy or integrity of any part of the work are appropriately investigated and resolved.

\section{Disclosure}

The authors report no conflicts of interest in this work.

\section{References}

1. Nagi SZ. The concept and measurement of disability. In: Berkowiz ED, editor. Disability Policies and Government Programs. New York, NY: Praeger; 1979:1-15.

2. National Center for Health Statistics. Health, United States, 2012: With Special Feature on Emergency Care. Hyattsville, MD: National Center for Health Statistics; 2012. Available from: http://www.ncbi.nlm.nih. gov/books/NBK148940/pdf/TOC.pdf. Accessed June 20, 2014.

3. Henry-Sanchez JT, Kurichi JE, Xie D, Pan Q, Stineman MG. Do elderly people at more severe activity of daily living limitation stages fall more? Am J Phys Med Rehabil. 2012;91(7):601-610.

4. Del Duca GF, Silva SG, Thumé E, Santos IS, Hallal PC. Predictive factors for institutionalization of the elderly: a case-control study. Rev Saude Publica. 2012;46(1):147-153.

5. Bakula MA, Kovacević D, Sarilar M, Palijan TZ, Kovac M. Quality of life in people with physical disabilities. Coll Antropol. 2011; 35(Suppl 2):247-253.

6. Cesari M, Onder G, Zamboni V, et al. Physical function and self-rated health status as predictors of mortality: results from longitudinal analysis in the ilSIRENTE study. BMC Geriatr. 2008;8:34-42.

7. Centers for Disease Control and Prevention. Self-reported increased confusion or memory loss and associated functional difficulties among adults aged $\geq 60$ Years -21 States, 2011. In: Morbidity and Mortality Weekly Report (MMWR), May 10, 2013. Atlanta, GA: Centers for Disease Control and Prevention. Available from: http://www.cdc.gov/ mmwr/preview/mmwrhtml/mm6218a1.htm. Accessed June 20, 2014.

8. Atkinson HH, Cesari M, Kritchevsky SB, et al. Predictors of combined cognitive and physical decline. J Am Geriatr Soc. 2005;53(7): 1197-1202.

9. Hairi NN, Cumming RG, Naganathan V, et al. Loss of muscle strength, mass (sarcopenia), and quality (specific force) and its relationship with functional limitation and physical disability: the Concord Health and Ageing in Men Project. J Am Geriatr Soc. 2010;58(11):2055-2062.

10. Nourhashémi F, Andrieu S, Gillette-Guyonnet S, et al. Is there a relationship between fat-free soft tissue mass and low cognitive function? Results from a study of 7,105 women. J Am Geriatr Soc. 2002;50(11):1796-1801.

11. Shin HY, Kim SW, Kim JM, Shin IS, Yoon JS. Association of grip strength with dementia in a Korean older population. Int $J$ Geriatr Psychiatry. 2012;27(5):500-505.

12. Burns JM, Johnson DK, Watts A, Swerdlow RH, Brooks WM. Reduced lean mass in early Alzheimer disease and its association with brain atrophy. Arch Neurol. 2010;67(4):428-433.

13. Sternäng O, Reynolds CA, Finkel D, Ernsth-Bravell M, Pedersen NL, Dahl Aslan AK. Factors associated with grip strenght decline in older adults. Age Ageing. Epub 2014 Nov 1.

14. MacDonald SW, DeCarlo CA, Dixon RA. Linking biological and cognitive aging: toward improving cgaracterizations of developmental time. J Gerontol B Psychol Sci Soc Sci. 2011;66(Suppl 1):i59-i70.

15. Nasreddine ZS, Phillips NA, Bédirian V, et al. The Montreal Cognitive Assessment, MoCA: a brief screening tool for mild cognitive impairment. J Am Geriatr Soc. 2005;53(4):695-699.
16. Galvin JE, Roe CM, Powlishta KK, et al. The AD8: a brief informant interview to detect dementia. Neurology. 2005;65(4):559-564.

17. Galvin JE, Roe CM, Coats MA, Morris JC. Patient's rating of cognitive ability: using the $\mathrm{AD} 8$, a brief informant interview, as a self-rating tool to detect dementia. Arch Neurol. 2007;64(5):725-730.

18. Galvin JE, Fagan AM, Holtzman DM, Mintun MA, Morris JC. Relationship of dementia screening tests with biomarkers of Alzheimer's disease. Brain. 2010;133(11):3290-3300.

19. Wilkins Ch, Roe CM, Morris JC. A brief clinical tool to assess physical function: the mini-physical performance test. Arch Gerontol Geriatr. 2010;50(1):96-100.

20. Reuben DB, Siu AL. An objective measure of physical function of elderly outpatients. The Physical Performance Test. J Am Geriatr Soc. 1990;38(10):1105-1112.

21. Guralnik JM, Simonsick EM, et al. A short physical performance battery assessing lower extremity function: association with self-reported disability and prediction of mortality and nursing home admission. J Gerontol. 1994;49(2):M85-M94.

22. Miller DK, Malmstrom TK, Andresen EM, et al. Development and validation of a short portable sarcopenia measure in the African American health project. J Gerontol A Biol Sci Med Sci. 2009;64(3): 388-394.

23. Rantanen T, Era P, Kauppinen M, Heikkinen E. Maximal isometric muscle strength and socioeconomic status, health, and physical activity in 75-year-old persons. J Aging Phys Act. 1994;2:206-220.

24. Rosenberg IH. Sarcopenia: origins and clinical relevance. J Nutr. 1997; 127(5 Suppl):990S-991S.

25. Baumgartner RN, Koehler KM, Gallagher D, et al. Epidemiology of sarcopenia among the elderly in New Mexico. Am J Epidemiol. 1998; 147(8):755-763.

26. Morley JE, Baumgartner RN, Roubenoff R, Mayer J, Nair KS. Sarcopenia. J Lab Clin Med. 2001;137(4):231-243.

27. Cruz-Jentoft AJ, Baeyens JP, Bauer JM, et al; European Working Group on Sarcopenia in Older People. Sarcopenia: European consensus on definition and diagnosis: Report of the European Working Group on Sarcopenia in Older People. Age Ageing. 2010;39(4): 412-423.

28. Yoshida D, Suzuki T, Shimada H, et al. Using two different algorithms to determine the prevalence of sarcopenia. Geriatr Gerontol Int. 2014; 14(Suppl 1):46-51.

29. Zigmond AS. The HADS: Hospital Anxiety and Depression Scale. Glasgow: N F E R Nelson Publishing Co; 1994.

30. Burnham KP, Anderson DR. Model Selection and Multimodel Inference: A Practical Information-Theoreic Approach. 2nd ed. New York, NY: Springer-Verlag Inc; 2002.

31. Janssen I, Heymsfield SB, Ross R. Low relative skeletal muscle mass (sarcopenia) in older persons is associated with functional impairment and physical disability. J Am Geriatr Soc. 2002;50(5):889-896.

32. Buchman AS, Wilson RS, Boyle PA, Bienias JL, Bennett DA. Grip strength and the risk of incident Alzheimer's disease. Neuroepidemiology. 2007;29(1-2):66-73.

33. van Kan GA, Cesari M, Gillette-Guyonnet S, Dupuy C, Vellas B, Rolland Y. Association of a 7-year percent change in fat mass and muscle mass with subsequent cognitive dysfunction: the EPIDOS-Toulouse cohort. J Cachexia Sarcopenia Muscle. 2013;4(3):225-229.

34. Van Kan GA. Epidemiology and consequences of sarcopenia. J Nutr Health Aging. 2009;13(8):708-712.

35. Clark BC, Manini TM. Functional consequences of sarcopenia and dynapenia in the elderly. Curr Opin Clin Nutr Metab Care. 2010;13(3): 271-276.

36. Studenski SA, Peters KW, Alley DE, et al. The FNIH Sarcopenia Project: rationale, study description, conference recommendations, and final estimates. J Gerontol A Biol Sci Med Sci. 2014;69(5): $547-558$.

37. Bandinelli S, Benvenuti E, Del Lungo I, et al. Measuring muscular strength of the lower limbs by hand-held dynamometer: a standard protocol. Aging (Milano). 1999;11(5):287-293. 
38. Xue QL, Walston JD, Fried LP, Beamer BA. Prediction of risk of falling, physical disability, and frailty by rate of decline in grip strength: the women's health and aging study. Arch Intern Med. 2011;171(12): 1119-1121.

39. Nilwik R, Snijders T, Leenders M, et al. The decline in skeletal muscle mass with aging is mainly attributed to a reduction in type II muscle fiber size. Exp Gerontol. 2013;48(5):492-498.

40. Short KR, Bigelow ML, Kahl J, et al. Decline in skeletal muscle mitochondrial function with aging in humans. Proc Natl Acad Sci US A. 2005;102(15):5618-5623.

41. Raji MA, Kuo YF, Snih SA, Markides KS, Peek MK, Ottenbacher KJ. Cognitive status, muscle strength, and subsequent disability in older Mexican Americans. J Am Geriatr Soc. 2005;53(9):1462-1468.

42. Roubenoff R. Exercise, sarcopenia, cognition, and mood. Nestle Nutr Workshop Ser Clin Perform Programme. 2002;6:151-159; discussion $160-162$.

43. Gonzalez-Freire M, de Cabo R, Studenski SA, Ferrucci L. The neuromuscular junction: aging at the crossroad between nerves and muscle. Front Aging Neurosci. 2014;6:

44. Wilkins CH, Roe CM, Morris JC, Galvin JE. Mild physical impairment predicts future diagnosis of dementia of the Alzheimer's type. $\mathrm{J} \mathrm{Am}$ Geriatr Soc. 2013;61(7):1055-1059.

45. Pratesi A, Tarantini F, Di Bari M. Skeletal muscle: an endocrine organ. Clin Cases Miner Bone Metab. 2013;10(1):11-14.

46. Schaap LA, Pluijm SM, Deeg DJ, Visser M. Inflammatory markers and loss of muscle mass (sarcopenia) and strength. Am J Med. 2006;119(6): 526. e9-e17.
47. Hull M, Strauss S, Berger M, Volk B, Bauer J. Inflammatory mechanisms in Alzheimer's disease. Eur Arch Psychiatry Clin Neurosci. 1996; 246(3):124-128.

48. Meng SJ, Yu LJ. Oxidative stress, molecular inflammation and sarcopenia. Int J Mol Sci. 2010;11(4):1509-1526.

49. Semba RD, Ferrucci L, Sun K, et al. Oxidative stress and severe walking disability among older women. Am J Med. 2007;120(12):1084-1089.

50. Mangialasche F, Polidori MC, Monastero R, et al. Biomarkers of oxidative and nitrosative damage in Alzheimer's disease and mild cognitive impairment. Ageing Res Rev. 2009;8(4):285-305.

51. Hsu YH, Liang CK, Chou MY, et al. Association of cognitive impairment, depressive symptoms and sarcopenia among healthy older men in the veterans retirement community in southern Taiwan: a cross-sectional study. Geriatr Gerontol Int. 2014;14(Suppl 1):102-108.

52. McLean RR, Shardell MD, Alley DE, et al. Criteria for clinically relevant weakness and low lean mass and their longitudinal association with incident mobility impairment and mortality: the foundation for the National Institutes of Health (FNIH) sarcopenia project. J Gerontol A Biol Sci Med Sci. 2014;69(5):576-583.

53. Liu CK, Leng X, Hsu FC, et al. The impact of sarcopenia on a physical activity intervention: the Lifestyle Interventions and Independence for Elders Pilot Study (LIFE-P). J Nutr Health Aging. 2014;18(1):59-64.
Clinical Interventions in Aging

\section{Publish your work in this journal}

Clinical Interventions in Aging is an international, peer-reviewed journal focusing on evidence-based reports on the value or lack thereof of treatments intended to prevent or delay the onset of maladaptive correlates of aging in human beings. This journal is indexed on PubMed Central, MedLine,

\section{Dovepress}

CAS, Scopus and the Elsevier Bibliographic databases. The manuscript management system is completely online and includes a very quick and fair peer-review system, which is all easy to use. Visit http://www.dovepress. com/testimonials.php to read real quotes from published authors. 